\title{
A Control Architecture for Grasp Strength Regulation in Myocontrolled Robotic Hands Using Vibrotactile Feedback: Preliminary Results
}

\author{
Roberto Meattini, Luigi Biagiotti, Gianluca Palli, Daniele De Gregorio and Claudio Melchiorri
}

\begin{abstract}
Nowadays, electric-powered hand prostheses do not provide adequate sensory instrumentation and artificial feedback to allow users voluntarily and finely modulate the grasp strength applied to the objects. In this work, the design of a control architecture for a myocontrol-based regulation of the grasp strength for a robotic hand equipped with contact force sensors is presented. The goal of the study was to provide the user with the capability of modulating the grasping force according to target required levels by exploiting a vibrotactile feedback. In particular, the whole human-robot control system is concerned (i.e. myocontrol, robotic hand controller, vibrotactile feedback.)

In order to evaluate the intuitiveness and force tracking performance provided by the proposed control architecture, an experiment was carried out involving four naïve ablebodied subjects in a grasping strength regulation task with a myocontrolled robotic hand (the University of Bologna Hand), requiring for grasping different objects with specific target force levels. The reported results show that the control architecture successfully allowed all subjects to achieve all grasping strength levels exploiting the vibrotactile feedback information. This preliminary demonstrates that, potentially, the proposed control interface can be profitably exploited in upper-limb prosthetic applications, as well as for non-rehabilitation uses, e.g. in ultralight teleoperation for grasping devices.
\end{abstract}

\section{INTRODUCTION}

The act of correctly grasping objects has been shown over decades to be a very complex task, since many degrees of freedom have to be activated in a simultaneous and coordinated manner, along with learning and percetion skills [1]. In this relation, the human hand presents remarkable capabilities in object manipulation, whereas, in contrast, the control of grasping devices such that to replicate human's proportional and fine physical interactions with objects is still a big challange in the scientific community [2].

This is also the case of human-robot interface systems. In this context, one of the most popular approaches of controlling robotic hands in human-in-the-loop scenarios is the use of myocontrol [3]. A myocontrolled robotic hand consists of the online extraction of control signals from muscle activity measurements (traditionally surface electromyography, sEMG [4]) in order to regulate the behavior of the grasping device. However, although sEMG signals present positive features to realize control interfaces, we always have to deal with their innate instability [5]. On the other hand, it has been shown how the providing of an artificial sensory feedback related to the grasp strength plays a role in improving the consistency of the commands generated by means of myocontrol during target grasping tasks [6]. Moreover, the

The authors are with the Department of Electrical, Electronic and Information Engineering, University of Bologna, 40126 Bologna, Italy presence of a grasping force feedback is one of the principal requirements in myocontrolled robotic hands for prosthetic purposes as a partial restoration of the amputees' sensory function. Nevertheless, none of the available prosthetic hands nowadays provide sensory feedback to the user about the interactions exchanged between robotic hand and objects ${ }^{1}$ [8].

In literature, researchers have used different non-invasive modalities to implement an intuitive feedback stimulation for the user, and most common methods provide patterned electrotactile, mechanotactile, or vibrotactile stimulation of the skin, in accordance with the general approach known as sensory substitution [9]. Here, we are interested in focusing on the vibrotactile modality (i.e., the use of vibration motors to deliver skin mechanical stimulations.) Note that vibrotactile feedback has been already studied in several works [10], [11], [12], and is not a recent approach at all.

In this work we did not want to answer the question if force feedback (in our application based on a vibrotactile device) can help the user during grasping operations. Many works on this topic have been proposed in the literature and the problem is still open [13]. The actual goal of this research activity was to investigate how to allow the user reproduce fine, target strength reference values exploiting vibrotactile feedback while grasping objects with a mycontrolled robotic hand. For this reason the main innovation of this article concerns the design of a complete human-robot control architecture, in which the user provides velocity commands to the hand, equipped with contact force sensors mounted on the fingertips, and receives an information that synthesizes the interaction force during grasping via the vibrotactile device.

In order to test the performance of the proposed control architecture, we involved four naïve able-bodied subjects in a simple experiment consisting of operating a myocontrolled robotic hand (the University of Bologna Hand, UB Hand), producing different target strength levels while grasping objects of different shape. The results show that all subjects successfully accomplished the required task for all objects and grasping force levels.

The paper is organized as follows: in Sec. II the devices used in this work are presented along with the concepts of the proposed control architecture; in Sec. III the protocol and results of the experiment are illustrated; finally, Sec. IV outlines the conclusions.

\footnotetext{
${ }^{1}$ A recent exception has to be mentioned: the VINCENTevolution2 system [7] equipped with a simple feedback of touch onset using a single vibration motor.
} 


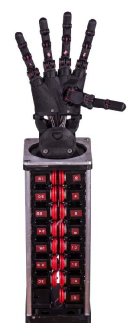

(a) The UB Hand

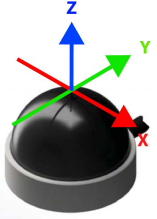

(b) Force sensor

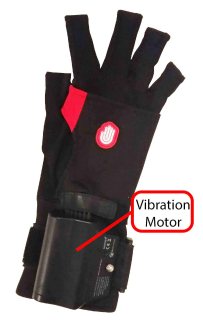

(c) Noitom Hi5 Glove.
Fig. 1. The UB Hand, contact force sensor and Hi5 Glove.

\section{MATERIALS AND METHODS}

\section{A. sEMG Setup and Acquisition}

The acquisition of the sEMG signals was perfomed by means of the biopotential wearable board Cerebro [14]. The data were sampled and streamed at $1 k H z$ to a nearby PC using an onboard Bluetooth interface. Two couples of differential low-cost surface electrodes (two sensors for each EMG channel) were placed on the skin in correspondence of the Flexor Digitorum Superficialis (FDS) and the Extensor Digitorum Communis (EDC) muscles. In order to use the sEMG for myocontrol (see next Subsec. II-D.1), the following filtering procedure was applied to the signals [15]: (i) a $50 \mathrm{~Hz}$ notch filter for powerline interference cancellation, (ii) a $20 \mathrm{~Hz}$ high-pass filter and, finally, (iii) the Root Mean Square (RMS) value was computed on a $200 \mathrm{~ms}$ window.

\section{B. UB Hand and Contact Force Sensors Description}

The robotic hand used within the present work was the UB Hand [16], see Fig. 1(a). The hand is a dexterous, anthropomorphic fully actuated grasping device, characterized by a particular actuation system that requires 5 coupled tendons for each finger of the hand (with 3 actuated DoF). In total, there are $n_{J}=15$ DoF actuated by $n_{m}=25$ tendons, each one driven by a Dynamixel RX-24F servomotor. The position control of the servomotors is based on a simple proportional control, that is

$$
\tau_{m}=K_{m}\left(\theta_{m}^{r e f}-\theta_{m}\right)
$$

where $\tau_{m} \in \mathbb{R}^{n_{m}}$ denotes the vector of the motor torques, $K_{m}=\operatorname{diag}\left(K_{m, 1}, \ldots, K_{m, i}, \ldots, K_{m, n_{m}}\right)$ is the proportional gain matrix, and $\theta_{m}^{r e f}, \theta_{m} \in \mathbb{R}^{n_{m}}$ are the reference and actual position of the motors, respectively. Note that by acting on the elements of the diagonal of $K_{m}$ it is possible to select the stiffness of each motor. At the joint control level, the three actuated joints of a finger are connected to 5 tendons driven by 5 servomotors, as described in [16]. The mapping between the joint and motor space is described by the linear relation

$$
\theta_{m}=H \theta_{J}, \quad \text { with } \quad H \in \mathbb{R}^{n_{m} \times n_{J}},
$$

where $\theta_{J} \in \mathbb{R}^{n_{J}}$ is the vector of the joint angles. Therefore, the reference value $\theta_{m}^{r e f}$ (see eq. (1)) can be computed according to eq. (2) once a desired joint configuration $\theta_{J}:=\theta_{J}^{r e f}$ is given. The computation of the joint reference configuration according to the control architecture presented in this work is shown in Sec. II-D.2.

Furthermore, the robotic hand is equipped with three semispherical contact force sensors (OMD-20-SE-40N Optoforce [17]), which measure the amplitude of forces along the $x, y$ and $z$ directions as shown in Fig. 1(b), namely $f_{x}, f_{y}, f_{z}$. The three sensors are placed on the fingertip of the thumb, index and middle fingers thanks to the presence of a 3D-printed custom support (see Fig. 5(a).) In this work, the norm of the force vector applied to the sensor is used, computed as $\left|f_{i}\right|=\sqrt{f_{x, i}^{2}+f_{y, i}^{2}+f_{z, i}^{2}}$, with $i=\{T, I, M\}$ referring to the thumb, index and middle fingers, respectively.

\section{Vibration Motor for the Vibrotactile Feedback}

The vibrotactile stimulation to the user of the myocontrolled robotic hand was provided by means of the vibration motor embedded on the wrist armband of the Noitom Hi5 glove [18] (see Fig. 1(c)), a wearable device for hand's finger motion tracking and feedback stimulation. Note that the motion tracking functionality of the glove is not used, and only the vibration motor was applied to the wrist of the subjects during the experimental session, as illustrated in Fig. 5(a). Specifically, this vibration motor can be controlled at the rate of $1 \mathrm{~Hz}$ by commanding the duration time of a vibration (at a given constant intensity) through a Bluetooth interface. Therefore, the modulation of the vibrotactile feedback stimulation was obtained by implementing a pulse-width modulation approach, in which an input signal $\nu \in[0,1]$ - namely, the normalized feedback signal - modulates the duty-cycle of the vibration motor activation signal (only two levels: high value for vibration, low value for no vibration) within a pulse period $T=1 s$. An example of a dutycyle modulation of the vibrotactile feedback stimulation is provided in Fig. 3.

\section{Control Architecture}

Preliminary Concepts: The realized control architecture is presented in the following, organized in the subsections Myocontrol, UB Hand Controller and Vibrotactile Feedback. For the mycontrol, a factorization approach based on Nonnegative Matrix Factorization (NMF [19]) was used, as illustrated in detail in Subsec. II-D.1. Note that the control by the user of different robotic grasp actions at the same time was not within the interests of the present work. In this relation, NMF has been successfully used for semi-unsupervised, human-like myocontrol based on muscle synergies related to user's opening/closing hand motions [20]. For the UB Hand controller, the grasp was realized using a myocontroldriven input signal for the regulation of the robotic hand joint velocities, combined with a postural synergy based approach [21]. Furthermore, also the measurements of the contact force sensors were exploited in the UB Hand controller, as outlined in Subsec. II-D.2. Finally, the vibrotactile feedback stimulation is exaplained in detail, reporting for the generation of the normalized feedback signal (see also Subsec. II-C.) The general scheme of the control architecture can be observed in Fig. 2. 


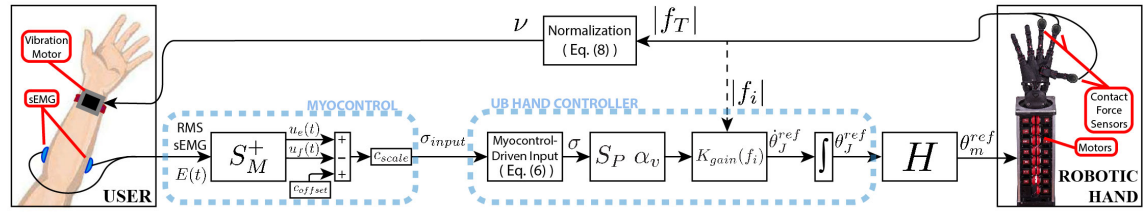

Fig. 2. Scheme of the control architecture for the grasp strength regulation.

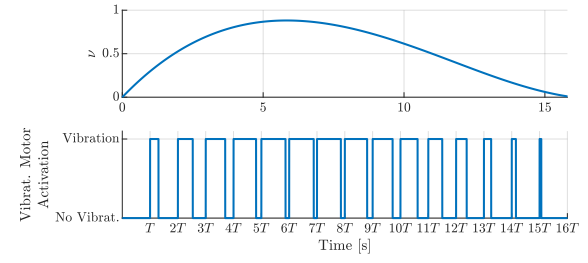

Fig. 3. Example of the duty-cycle modulation of the vibrotactile feedback stimulation.
1) Myocontrol: In order to obtain a control signal proportional to the closure level of the user's hand - namely the hand closure level $\sigma_{\text {input }}$ - the NMF algorithm ${ }^{2}$ was applied to forearm's sEMG signals to determine the muscular synergy matrix $S_{M}$ related to hand motions. In detail, the estimation of $S_{M}$ was carried out by means a short calibration phase. In particular, the user had to perform a simple specific motion: open and close her hand (two times). At the same time, the sEMG signals were acquired to build a matrix $E_{\text {offline }} \in \mathbb{R}^{n_{E} \times n}$, where $n_{E}$ is the number of sEMG channels (in our case $n_{E}=2$, see Subsec. IIA) and $n$ is the number of samples collected. Then the NMF algorithm was applied for the computation of the muscular synergy matrix $S_{M} \in \mathbb{R}^{n_{E} \times n_{U}}$, according to the equation $E_{\text {offline }} \approx S_{M} U_{\text {offline }}$, with $U \in \mathbb{R}^{n_{U} \times n}$ the socalled offline neural drives matrix, and $n_{U}$ the number of supraspinal neural drives (in our case $n_{U}=2$ ). For further details on the application of NMF for the estimation of the muscular synergy matrix during hand closure motions, refer to our previous work [20].

Once obtained the matrix $S_{M}$, the online computation of the neural drives could be performed according to

$$
U(t)=S_{M}^{+} E(t),
$$

where $U(t)=\left[u_{e}(t) u_{f}(t)\right]^{\mathrm{T}} \in \mathbb{R}^{2}$ is the vector of the instantaneous values of the neural drives, $S_{M}^{+}$is the pseudoinverse matrix of $S_{M}$ and $E(t)=\left[e_{1}(t) e_{2}(t)\right]^{\mathrm{T}} \in \mathbb{R}^{2}$ is the vector of the instantaneous values of the sEMG channels.

Thereafter, the hand closure level was obtained from the estimated neural drives as

$$
\sigma_{\text {input }}=c_{\text {scale }}\left(u_{e}(t)-u_{f}(t)+c_{o f f s e t}\right)
$$

where $c_{\text {scale }}$ and $c_{\text {offset }}$ are proper constants in order to map $\sigma_{\text {input }}$ in the range $[-0.5,0.5]$. Then, this signal is connected, as an input, to the UB Hand controller (see Fig. 2.)

2) UB Hand Controller: Also referring to some notation previously introduced in Subsec. II-B, let us define $\dot{\theta}_{J}^{r e f} \in$ $\mathbb{R}^{n_{J}}$ the vector of the robotic hand joint velocities. In par-

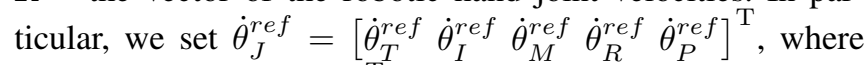
$\dot{\theta}_{i}^{r e f}=\left[\begin{array}{ccc}\dot{\theta}_{i, 1}^{r e f} & \dot{\theta}_{i, 2}^{r e f} & \dot{\theta}_{i, 3}^{r e f}\end{array}\right]^{\mathrm{T}}$ is the vector of the joint velocities of a single finger, with $i=\{T, I, M, R, P\}$ indicating thumb, index, middle, ring and pinkie fingers, respectively. Then, the instantaneous joint reference configuration $\theta_{J}^{\text {ref }}$

\footnotetext{
${ }^{2}$ Given a nonnegative matrix $A \in \mathbb{R}^{m \times n}$ (a matrix whose elements are all non negative), the product $W H$ is called NMF of $A$ if nonnegative matrices $W \in \mathbb{R}^{m \times k}$ and $H \in \mathbb{R}^{k \times n}$, with $k<\min (m, n)$, are found such that the functional $f(W, H)=\frac{1}{2}\|A-W H\|_{F}^{2}$ is minimized [19].
}

was defined according to the concept of postural synergies, and given by the velocity control law

$$
\dot{\theta}_{J}^{r e f}=K_{\text {gain }} S_{P} \alpha_{v} \sigma .
$$

In eq. (5), $\sigma$ is the myocontrol-driven control input given by

$$
\sigma= \begin{cases}\sigma_{\text {input }}-\bar{\sigma}_{1}, & \text { if } \sigma_{\text {input }} \leq \bar{\sigma}_{1} \\ 0, & \text { if } \bar{\sigma}_{1}<\sigma_{\text {input }}<\bar{\sigma}_{2}, \\ \sigma_{\text {input }}-\bar{\sigma}_{2}, & \text { if } \sigma_{\text {input }} \geq \bar{\sigma}_{2}\end{cases}
$$

where $\bar{\sigma}_{1}$ and $\bar{\sigma}_{2}$ are two constant threshold values with $\bar{\sigma}_{2}>\bar{\sigma}_{1}$ (in the experimental session reported in Sec. III, $\bar{\sigma}_{1}=-0.1$ and $\bar{\sigma}_{1}=0.1$ ), and $\sigma_{\text {input }}$ is provided online - modulated by the hand opening/closing motions of the user - in accordance with eq. (4). $\alpha_{v} \in \mathbb{R}^{3}$ is a versor in the postural synergy subspace defined as $\alpha_{v}=$ $\left(\alpha_{\text {closed }}-\alpha_{\text {open }}\right) /\left(\left\|\alpha_{\text {closed }}-\alpha_{\text {open }}\right\|\right)$, being $\alpha_{\text {open }}$ and $\alpha_{\text {closed }}$ the hand postures in the synergy subspace closest to the hand totally open and closed postures in the joint space, respectively. $S_{P} \in \mathbb{R}^{n_{J} \times n_{S}}$ is the postural synergy matrix (computed as in our previous work [22]), where $n_{S}=3$ is the number of eigenpostures [22] taken into consideration. Finally, $K_{\text {gain }} \in \mathbb{R}^{n_{J} \times n_{J}}$ is a diagonal gain matrix depending on the contact force of each finger, defined as

$$
K_{\text {gain }}=\operatorname{diag}(\underbrace{K_{T}, \ldots, K_{T}}_{4 \text { times }}, \ldots, \underbrace{K_{i}, \ldots, K_{i}}_{4 \text { times }}, \ldots, \underbrace{K_{P}, \ldots, K_{P}}_{4 \text { times }}),
$$

where $i=\{T, I, M, R, P\}$ denotes the different robotic hand fingers and

$$
K_{i}= \begin{cases}\bar{K}\left(\frac{1-\gamma}{\gamma} \frac{\left|f_{i}\right|}{\bar{f}}+1\right), & \text { if }\left|f_{i}\right|<\bar{f} \text { for } i=\{T, I, M\} \\ \frac{\bar{K}}{\gamma}, & \text { if }\left|f_{i}\right| \geq \bar{f} \text { for } i=\{T, I, M\}, \\ 0, & \forall\left|f_{i}\right| \quad \text { for } i=\{R, P\}\end{cases}
$$

in which $\left|f_{i}\right| \in \mathbb{R}^{+}$is the norm of a single finger force sensor, $\bar{f}$ is a constant threshold (empirically determined) equal to the minimum force value to consider a fingertip in contact with an object, $\bar{K}$ is the control gain when there is no contact with the object and $\gamma$ is a factor to scale the gain $\bar{K}$ to the lower value $\bar{K} / \gamma$ when a specific finger comes into contact. Note that $\gamma$ produces a reduction of the gain $K_{i}$ that, in turn, determines the extent of the contribution of the user command (i.e. the myocontrol-driven input $\sigma$, see eq. (6)) to the variation of the hand joint velocities, having a twofold reason: $(i)$ the motion of the finger that came into contact with the object is slowed down, allowing 


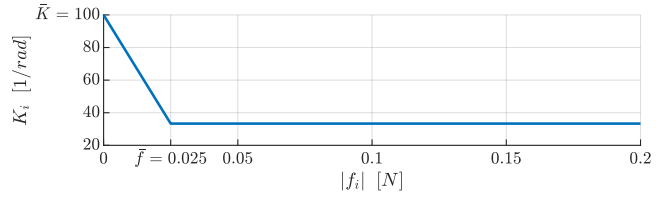

Fig. 4. Values of $K_{i}$ as a function of the norm of the contact force. TABLE I

OBJECTS USED IN THE EXPERIMENT.

\begin{tabular}{|c|c|c|c|} 
Object Type & Box & Cylinder & Sphere \\
\hline Size [mm] & $88 \times 54 \times 54$ & $100 \times 75 \times 75$ & 65 (radius)
\end{tabular}

for an adaptation of the grasp to the object shape, and (ii) the capability of the user in finely modulating the grasp strength is highly increased, thanks to the possibility of providing small adjustment to the value of the robotic hand joint reference (see eq. (5)). Finally, note that, for $i=\{R, P\}$, $K_{i}$ was set to zero because in this study only tripodal grasps were considered. Fig. 4 depicts the values of $K_{i}$ (for $i=\{T, I, M\}$ ) as a function of $f_{i}$. Eqs. (5)-(7) are summarized in the block diagram of Fig. 2.

3) Vibrotactile Feedback: The vibrotactile stimulation was provided to the user on the basis of the normalized feedback signal $\nu$ (see Subs. II-C), which was a function of the estimated internal force of the grasp, i.e. the forces exterted at the contact points that present zero resultant forces on the object. Formally, these forces are defined as the force vector which spans the null space of the so-called grasp matrix (often denoted by $G$ ) [23]. For precison tripodal grasps, the thumb fingertip is in contact in an opposite location with respect to the index and middle fingertips, and its contact force must be counteracted by the other two fingers and viceversa, therefore representing an approximated measure of the applied internal force. In line with the concept illustrated, the force exerted by the thumb was used to approximate the internal force, and the normalized force signal was given as

$$
\nu=\left\{\begin{array}{lll}
\frac{|| f_{T}\left|-\bar{f}_{T}\right|}{c_{\text {norm }}}, & \text { if } & || f_{T}\left|-\bar{f}_{T}\right| \leq c_{\text {norm }} \\
0, & \text { if } & || f_{T}\left|-\bar{f}_{T}\right| \leq \beta \\
1, & \text { if } & || f_{T}\left|-\bar{f}_{T}\right|>c_{\text {norm }}
\end{array},\right.
$$

where $c_{n o r m}$ is a normalization constant and $\beta$ denotes an error band for which the tracking of $\bar{f}_{T}$ is considered achieved (in our experiment $\beta=0.03$, see Subsec. III-B). Therefore, the combination of eq. (8) with the duty-cycle modulation of the vibration motor (see Subsec. II-C and Fig. 3) generated the vibrotactile stimulation feedback. Note that, since the vibrotactile feedback reflects the absolute value of the error between estimated and target grasp strength, during the experiment reported in Sec. III the user was assisted by a screen informing about the sign of the error, see Fig. 5(b) (whereas the extent of the error was available only through the vibrotactile stimulation).

\section{EXPERIMENT AND RESULTS}

In order to test the capability of a user to exploit the proposed control architecture, an experiment was conducted in-

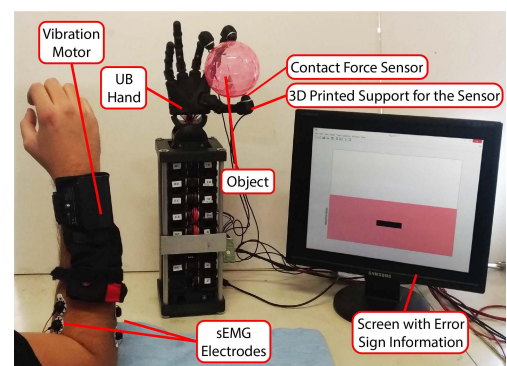

(a) Overview.
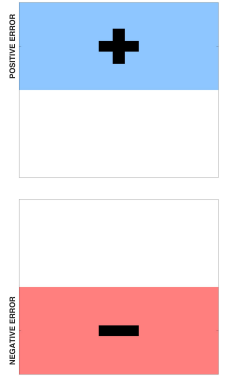

(b) $\left|f_{T}\right|-\bar{f}_{T}$ graphical information.
Fig. 5. The experimental setup for the grasp strength regulation experiment.

volving four naïve able-bodied subjects. The experiment was performed in accordance with the Declaration of Helsinki.

\section{A. Experimental Setup and Protocol}

Fig. 5(a) shows the setup organization for the experimental session. The subjects were four men between 25 and 35 years old (namely S1, S2, S3, S4), and none of them experienced myocontrol before. During the experiment, each subject was seated in front of a desk equipped with the UB Hand (described in Subsec. II-B) and a screen to provide the grasp strength error sign information (see Subsec. II-D.3). A couple of differential sEMG electrodes was placed in proximity of the FDS and EDC muscles' belly (see Susec. II-A), and the vibration motor described in Subsec. II-C was fixed on the forearm close to the wrist.

First of all, during the myocontrol training session, the subject had to fully open and close his hand for two times while the sEMG signals were recorded. After that, the system automatically computed the pseudo-inverse of the muscular synergy matrix $S_{M}^{+}$and the scaling constants as described in Subsec. II-D.1. At this point the myocontrol was calibrated and ready to be used. Thereafter, the grasping strength regulation task took place. The myocontrol was connected to the UB Hand, according to Subsec. II-D.2, and the subject received the vibrotactile feedback as described in Subsec. IIC and II-D.3. Specifically, at the beginning the subject had to keep the robotic hand fully open, until the experimenter handed out the first object. At this point the subject had to close the robotic hand to perform a tripodal grasp, see Fig. 5(a). Here, the experimenter released the object and the subjects had to regulate - by means of myocontrol - the grasp strength, according to three arbitrary reference levels. In detail, during the grasping, the first required strength level was represented (according to Subsec. II-D.3) by a thumb force $\bar{f}_{T}=0.2 N$, then varying to $\bar{f}_{T}=0.4 N$, and finally to $\bar{f}_{T}=0.6 N$. These three target values were chosen, in this preliminary experiment, with empirical method in order to allow a fine grasp strength regulation. Each of the grasping strength level had a duration of approximately $100 \mathrm{~s}$, and changed during the grasp, i.e., without the releasing of the object by the subject. In the end, the experimenter vocally instructed the subject to release the object (i.e., open the robotic hand) and took the object back with her. Three different objects were used in the experiment: namely the 

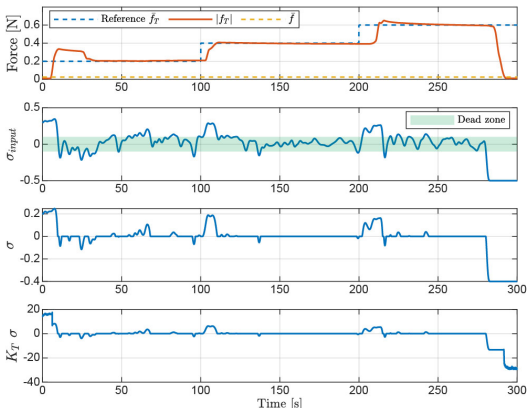

Fig. 6. Signals of the strength regulation with the Box object (sbj. S4).

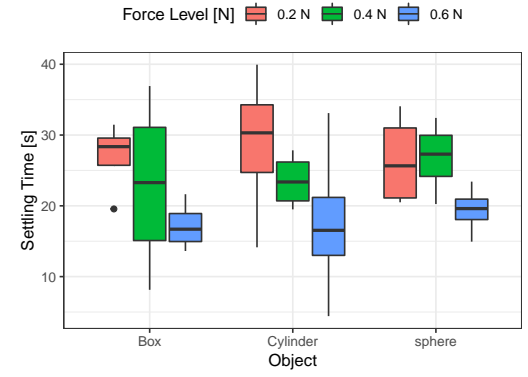

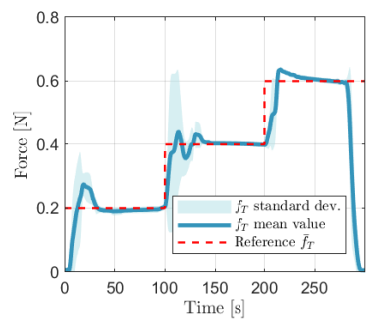

(a) Box object.

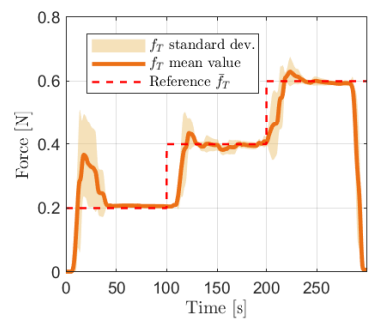

(b) Cylinder object.

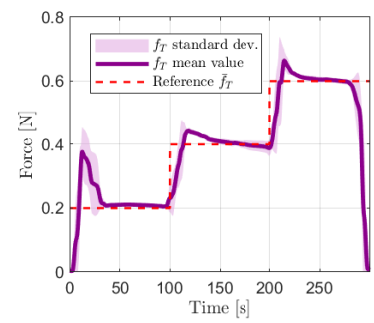

(c) Sphere object.

Fig. 7. Mean and standard deviation of the regulated grasp strength, computed over the subjects.
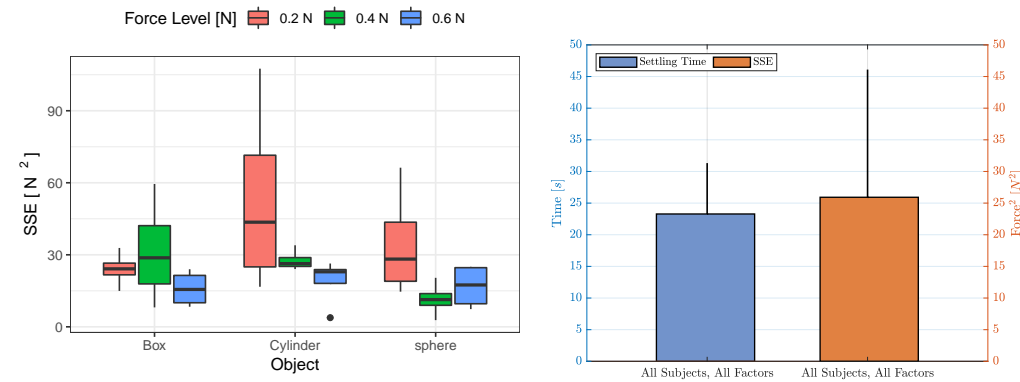

(a) Settling time grouped by Object, for the three (b) SSE grouped by Object, for the three possible (c) Bar graph of the data grouped by settling possible levels of Strength Reference Level. levels of Strength Reference Level. time and SSE.

Fig. 8. Grouped data based on the factors for the ANOVA and on the metrics settling time and SSE.

Box, Cylinder and Sphere (supplied in this order), whose dimensions and figures are reported in Tab. I. Three different regulation tasks with the three strength levels were carried out for the three different objects. The subject rested 10 minutes after each single task.

\section{B. Grasp Strength Regulation Results}

Fig. 6 shows the resulting signals for the grasping stength regulation task of one of the subject (S4) with the Box object. Looking at the figure, in the top graph it is possible to see how the grasp strength - approximately represented by the thumb force $\left|f_{T}\right|$ (see Subsec. II-D.3) - was succefully modulated in order to reach the three different reference levels, after presenting undershoot/overshoot behaviour and different settling times at the beginning of each strength level. Note that we considered the tracking of a reference level succefully achieved when $\left|f_{T}\right|$ entered within an error band equal to $\pm 5 \%$ of the higher stength reference level $(0.6 N)$, i.e. the range $\left[\bar{f}_{T}-0.03, \bar{f}_{T}+0.03\right]$, and remained in this range until a new strength reference level was required (or until the experimenter instructed the subject to release the object, as at the time instant around 230s in Fig. 6.) Accordingly, the settling time is intended as the time to reach the successful tracking condition, once a reference strength level was given to the subject. Focusing now, in Fig. 6, to the graph of the hand closure level $\sigma_{\text {input }}$ (see Subsec. IID.1), it is possible to observe how this signal was intuitively regulated for force increase (time instants around $0 s, 100 s$, $200 s$ ), or for force decrease (time instant around $30 s, 290 s$ ). Indeed, this is clearly reflected in the graph plotting $\sigma$, the myocontrol-driven signal given by eq. (6), where we can see non-zero values only outside of the $\sigma_{r e f}$-related deadzone. Finally, the bottom graph of Fig. 6 illustrates how $\sigma$ was modulated by $K_{\text {gain }}$ : when $\left|f_{T}\right|<\bar{f}$ (orange dashed line in the top graph of Fig. 6), $K_{T}$ assumed higher values (see the case\#1 of eq. (7)), therefore also determining higher values of $K_{T} \sigma$ compared to when $f_{T} \geq \bar{f}$ (i.e. contact with the object) with $K_{T}$ assuming lower values (equal to $\bar{K} / \gamma$ according to case\#2 of eq. (7).) This shows how the subject had a lesser (greater) incidence on the increasing/decreasing of the grasp strength after (before) the fingertips came in contact with the object, due to a finer (faster and coarse) modulation of the robotic hand joint velocity references.

The behaviour shown in Fig. 6 was replicated by all the subjects, that successfully regulated the force for all the strength reference levels. This is reported in Fig. 7 in terms of mean and standardard deviation values of the regulated grasp strength, computed over the subjects. Furthermore, Fig. 7 also shows - on average over the subjects - how the tracking error of $\bar{f}_{T}$ increased during the transients (defined as the temporal portion between the setting of a strength reference level and the related settling time of $\left|f_{T}\right|$ ), showing also a greater variability in the performance among subjects.

In this relation, two metrics regarding only to the transient stages were statistically analyzed: the settling time and the Sum of Squared Errors (SSE). Therefore, a two-way repeated measure Analysis of Variance (ANOVA) was conducted for each of the two transient-related metrics. In the ANOVA, the two investigated factors were Object (Box, Cylinder or Sphere) and Strength Reference Level $(0.2 N, 0.4 N$ and $0.6 N)$, and the statistical significance was set to $p<.05$. The 
related boxplots of the different data groups can be found in Fig. 8. The Mauchly's test was performed to check the assumption of sphericity.

Considering first the settling time metric, the Mauchly's test indicated that the assumption of sphericity had been violated for the interaction effect between the strength level and the type of object, $W=2.4 \cdot 10^{-17}, p<.001$. Therefore the degree of freedom was corrected using the GreenhouseGeisser estimate of sphericity. All effects are reported as nonsignificant, $F(2,6)=3.55$ and $p>.05$ for the main effect of the strength level, $F(2,6)=0.24$ and $p>.05$ for the main effect of the type of object, and $F(1.58,2.38)=0.14$ and $p>.05$ for the interaction effect. For the SSE metric, Mauchly's test indicated that all effects had not violate the assumption of sphericity. As for the settling time metric, all effects are reported as non-significant, $F(2,6)=2.59$ and $p>.05$ for the main effect of the strength level, $F(2,6)=1.58$ and $p>.05$ for the main effect of the type of object, and $F(4,12)=1.95$ and $p>.05$ for the interaction effect. Therefore, the ANOVA revealed that the support of the experimental data is weak to make statistical conclusions about the main and interaction effects of the factors Object and Strength Reference Level, and therefore we cannot draw significant interpretations in this specific sense. Consequently, as usual in the case of non-significant factors, the related data is pooled together for the settling time and SSE metrics, as can be observed in the bar graph of Fig. 8(c), reporting for a mean settling time of $23.28 \mathrm{~s}$ and a mean SSE of $25.92 N^{2}$ computed over the four subjects involved in the experiment.

\section{CONCLUSIONS}

In this article a control architecture for the online regulation of the grasp strength of a myocontrolled robotic hand has been presented. In particular, a specific vibrotactile feedback stimulation on the basis of an approximation of the internal force of the grasp was provided to the user. The experimental results reported in Subsec. III-B show that all the subjects intuitively and successfully reached three target strength values (Fig. 7). In particular, during the force reaching transients, they presented a mean settling time of $23.28 \pm 8.04 \mathrm{~s}$ and a mean SSE of $25.92 \pm 20.17 N^{2}$. Upcoming investigations will be focused on several aspect. Firstly, the design of additional vibrotactile feedback modulations, with the aim of improving the user sensory awareness. Furthermore, a decrease of the settling time will be concerned, searching for more appropriate solutions both in the interface and control of the robotic hand. Finally, more advanced experimental protocols will be employed, involving trans-radial amputed subjects in the test of the myocontrolled robotic system.

\section{REFERENCES}

[1] C. L. MacKenzie and T. Iberall, The grasping hand. Elsevier, 1994, vol. 104.

[2] M. Santello, M. Bianchi, M. Gabiccini, E. Ricciardi, G. Salvietti, D. Prattichizzo, M. Ernst, A. Moscatelli, H. Jörntell, A. M. Kappers et al., "Hand synergies: integration of robotics and neuroscience for understanding the control of biological and artificial hands," Physics of life reviews, vol. 17, pp. 1-23, 2016.
[3] R. Merletti, M. Aventaggiato, A. Botter, A. Holobar, H. Marateb, and T. M. Vieira, "Advances in surface emg: recent progress in detection and processing techniques," Critical Reviews ${ }^{T M}$ in Biomedical Engineering, vol. 38, no. 4, 2010.

[4] R. Meattini, M. Nowak, C. Melchiorri, and C. Castellini, "Towards Improving Myocontrol of Prosthetic Hands : a Study on Automated Instability Detection," 2018.

[5] E. A. Biddiss and T. T. Chau, "Upper limb prosthesis use and abandonment: a survey of the last 25 years," Prosthetics and orthotics international, vol. 31, no. 3, pp. 236-257, 2007.

[6] A. Ninu, S. Dosen, S. Muceli, F. Rattay, H. Dietl, and D. Farina, "Closed-loop control of grasping with a myoelectric hand prosthesis: Which are the relevant feedback variables for force control?" IEEE Transactions on Neural Systems and Rehabilitation Engineering, vol. 22, no. 5, pp. 1041-1052, 2014.

[7] (2019) Vincent systems gmbh vincentevolution 2. [Online]. Available: http://vincentsystems.de/en/prosthetics/vincent-evolution-2/

[8] N. Jiang, S. Dosen, K.-R. Muller, and D. Farina, "Myoelectric control of artificial limbs-is there a need to change focus?[in the spotlight]," IEEE Signal Processing Magazine, vol. 29, no. 5, pp. 152-150, 2012.

[9] P. Bach-y Rita and S. W. Kercel, "Sensory substitution and the humanmachine interface," Trends in cognitive sciences, vol. 7, no. 12, pp. 541-546, 2003.

[10] C. Cipriani, F. Zaccone, S. Micera, and M. C. Carrozza, "On the shared control of an emg-controlled prosthetic hand: analysis of userprosthesis interaction," IEEE Transactions on Robotics, vol. 24, no. 1, pp. 170-184, 2008.

[11] C. Pasluosta, H. Tims, L. Chiu et al., "Slippage sensory feedback and nonlinear force control system for a low-cost prosthetic hand," Am. J. Biomed. Sci, vol. 1, no. 4, pp. 295-302, 2009.

[12] I. Saunders and S. Vijayakumar, "The role of feed-forward and feedback processes for closed-loop prosthesis control," Journal of neuroengineering and rehabilitation, vol. 8, no. 1, p. 60, 2011.

[13] T. A. Rohland, "Sensory feedback in upper limb prosthetic systems," Inter Clinic Information Bulletin, vol. 13, no. 1, pp. 1-4, 2013.

[14] S. Benatti, B. Milosevic, F. Casamassima, P. Schönle, P. Bunjaku, S. Fateh, Q. Huang, and L. Benini, "Emg-based hand gesture recognition with flexible analog front end," in 2014 IEEE Biomedical Circuits and Systems Conference (BioCAS) Proceedings. IEEE, 2014, pp. 5760.

[15] R. Meattini, S. Benatti, U. Scarcia, L. Benini, and C. Melchiorri, "Experimental evaluation of a semg-based human-robot interface for human-like grasping tasks," in 2015 IEEE International Conference on Robotics and Biomimetics (ROBIO). IEEE, 2015, pp. 1030-1035.

[16] C. Melchiorri, G. Palli, G. Berselli, and G. Vassura, "Development of the ub hand iv: Overview of design solutions and enabling technologies," IEEE Robotics \& Automation Magazine, vol. 20, no. 3, pp. 72-81, 2013.

[17] (2019) Onrobot optoforce omd-20-se-40n. [Online]. Available: https://onrobot.com/en

[18] (2019) Noitom hi5 vr glove. [Online]. Available: https://www.noitom.com/solutions/hi5-vr-glove

[19] M. W. Berry, M. Browne, A. N. Langville, V. P. Pauca, and R. J Plemmons, "Algorithms and applications for approximate nonnegative matrix factorization," Computational statistics \& data analysis, vol. 52, no. 1, pp. 155-173, 2007.

[20] R. Meattini, S. Benatti, U. Scarcia, D. De Gregorio, L. Benini, and C. Melchiorri, "An sEMG-Based Human-Robot Interface for Robotic Hands Using Machine Learning and Synergies," IEEE Transactions on Components, Packaging and Manufacturing Technology, vol. 8, no. 7, pp. 1149-1158, 2018.

[21] M. Santello, M. Flanders, and J. F. Soechting, "Postural Hand Synergies for Tool Use," The Journal of Neuroscience, vol. 18, no. 23, pp. 10105-10115, 1998. [Online]. Available: http://www.jneurosci.org/lookup/doi/10.1523/JNEUROSCI.1823-10105.1998

[22] F. Ficuciello, G. Palli, C. Melchiorri, and B. Siciliano, "Experimental evaluation of postural synergies during reach to grasp with the ub hand iv," in 2011 IEEE/RSJ International Conference on Intelligent Robots and Systems. IEEE, 2011, pp. 1775-1780.

[23] R. M. Murray, A mathematical introduction to robotic manipulation. CRC press, 2017. 